\title{
An Explicit Expression for the Korteweg-de Vries Hierarchy
}

\author{
R. SCHIMmING
}

Für die Korteweg-de Vries-Hierarchic von solitonischen partiellen Differentialgleichungen wird eine bisher nicht bekannte explizite Darstellung hergeleitet.

Выводится явное и ло сих пор неизвестне прсдытавление для иерархи Кортевегаде Фриза солцтоновых дифференццальных ураннений.

An explicit representation for the Korteweg-de Vries hierarchy of solitonic partial differential equations is derived which has not been known before.

\section{Introduction}

A nonlinear partial differential equation is called solitonic if it admits

- particle-like solutions, the so-called solitoris;

- Bäclilund transformations;

- a Lax and/or prolongation representation;

- application of, the inverse scattering method;

- infinitely many conservation laws.

The typical solitonic equations naned after Korteweg-de Vries, Burgers, Boussinesq; Kadomtsev-Petviashvili, ... have been discovered as physical models. The deeper mathematical reasons for their highly peculiar behaviour were difficult to recognize: Some progress has been achieved by extending a "seed equation" to a "hierarchy", that means to an infinite sequence of related solitonic equations of increasing order. The hierarchies considerably enlarge the reservoir of differential equations in soliton theory.

P. D. LAX [8] proposed to extend the Korteweg-de Vries equation

$$
u_{t}=6 u u_{x}+u_{x x x}
$$

for a function $u=u(x, t)$ of position $x$ and time $t$ to a hierarchy

$$
\begin{aligned}
& u_{t}=u_{3}+6 u u_{1}, \\
& u_{t}=u_{5}+10 u u_{3}+20 u_{1} u_{2}+30 u^{2} u_{1}, \\
& u_{t}=u_{7}+14 u u_{5}+\cdots,
\end{aligned}
$$

The general law for this

$$
u_{t}=\frac{\partial}{\partial x} \cdot G_{n}[u] \quad(n=2,3, \ldots)
$$


usually is described by recursion relations for the differential polynomials $G_{n}=G_{n}[u]$ $(n=1,2, \ldots)$. (A differential polynomial in $u$ is a polynomial in $u, u_{1}, u_{2}, \ldots$ with constant coefficients and without an absolute term.) I. M. GEL'FAND and L. A. Dikij [16] presented formulas for the $G_{n}$ which become explicit when certain multiple integrals or generating functions, respectively, are evaluated. The purpose of this paper is to derive a fully explicit representation for the right-hand sides of the Korteweg-de Vries hierarchy, namely

$$
\begin{aligned}
& n ![(2 n-1) !]^{-1} G_{n}[\dot{u}] \\
= & \sum\left[\left(q_{2}+n-1\right)\left(q_{3}+n-2\right) \ldots\left(q_{n}+1\right)\right]^{-1} \\
& \times c\left(0, q_{2}\right) c\left(q_{2}, \dot{q}_{3}\right) \ldots c\left(q_{n-1}, q_{n}\right) u_{-q_{2}} u_{q_{2}-q_{3}} \ldots u_{q_{n-1}-q_{n}} u_{q_{n}}
\end{aligned}
$$

We use the notations

$$
u_{t}=\frac{\partial u}{\partial t}, \quad u_{1}=u_{x}=\frac{\partial u}{\partial \dot{x}}, \quad u_{p}=u_{x x \ldots x}=\frac{\partial^{p} u}{\partial x^{p}} \quad(p \geqq 2)
$$

and also formaliy

$$
u_{0}=u, \quad u_{-1}=0, \quad u_{-2}=1 .
$$

The numerical coefficients above are given by

$$
c(p, q)=\left(\begin{array}{l}
p \\
q
\end{array}\right)+\delta_{q}^{p+2} \text { for integers } p, q \geqq 0
$$

and the sum runs over all integers $q_{2}, q_{3}, \ldots, q_{n}$ such that

$$
0 \leqq q_{2} \leqq 2,0 \leqq q_{3} \leqq q_{2}+\dot{2}, \ldots, 0 \leqq q_{n} \leqq q_{n-1}^{-}+2 .
$$

In $\S 1$ we develop the definition of the sequence $\left(G_{n}\right)=\left(G_{n}[u]\right)_{n \geqq 0}$ and introduce the Korteweg-de Vries hierarchy through lax pairs. The calculation behind th is has been done essentially by J. L. BurchNat. and T. W. Chausdy [2] as early as in 1922. 'Their priority is an interesting historical fact we would like to emphasize. The $\S 2$ is devoted to the proof of our formula. To be precise, a somewhat more general formula concerning Hadamard's coefficients to the one-dimensional Schrödinger operator is established. Use of the Minakshisundaram-Pleijel asynptotic expansion of the fundamental solution to the heat equation is made. In $\S 3$ we present, for the sake of completeness, additional properties of the $G_{1}, G_{2}, \ldots$ and of differential polynomials which are closely related to the $G_{1}, G_{2}, \ldots$

\section{$\$ 1$ Definition of the Korteweg-de Vries hierarchy *}

The construction starts with the one-dimensional Schrödinger equation (sometimes also called Sturm-Liouville equation)

$$
\left(L+k^{2}\right) y=y^{\prime \prime}+\left(u+k^{2}\right) y=0 .
$$

Here $L=D^{2}+u$ is the one-dimensional Schrödinger operator; $D=\partial / \partial x$; the energy constant $k$ is assumed to be real and nonzero; the potential $u=u(x)$ is assumed to be real-valued and smooth in some interval. (An additional dependence 
on a time $t$ is introduced later.) Using standard arguments from the literature (cf., e.g., $[17,18])$ one can construct a formal solution to (1.1) of the form

$$
\therefore y=\mathrm{e}^{1 k x} \sum_{n=0}^{\infty} z_{n}(2 \mathrm{i} k)^{-n}
$$

with real coefficients $z_{0}=1, z_{1}, z_{2}, \ldots$ From it we build up

$$
G:=k^{-1} y \bar{y}=: 4 \sum_{n=0}^{\infty} G_{n}(-1)^{n}(2 k)^{-2 n-1}
$$

('The power series may converge or not; in fact they serve' as generating functions for the sequences of their coefficients.) Here the $G_{n}$ are again real and $G_{0}=1 / 2$. Considering the first integral

$$
y^{\prime} \bar{y}-y \bar{y}^{\prime}=\text { const. }=2 \mathrm{i} k,
$$

we obtain from (1.1) the nonlinear second-order equation

$$
2 G G^{\prime \prime}-G^{\prime 2}+4\left(u+k^{2}\right) G^{2}=4 \text {. }
$$

Differentiation with respect to $x$ produces the linear third-order equation

$$
G^{\prime \prime \prime}+4\left(u+k^{2}\right) G^{\prime \prime}+2 u^{\prime} G=0 .
$$

Equivalent to this, the sequence $\left(G_{n}\right)_{n \geqq 0}$ obeys a differential-recursion equation system which has been named after $A$. Lenard $[3,9,10]$ :

$$
G_{n+1}^{\prime}=\dot{G}_{n}^{\prime \prime \prime}+4 u G_{n}^{\prime}+2 u^{\prime} G_{n}, \quad G_{0}:=1 / 2 .
$$

- The next step will be the construction of certain linear differential operators $A_{n}$ with respect to $x$. Note that we do not notationally distinguish between a function and the multiplication operator (or linear differential operator of zero order) defined by it.

Proposition 1.1: The operator-valued formal power series $A$ in $(2 k)^{-1}$ defined by

$$
4 A=\left(G^{\prime \prime}-2 G D\right)\left(L+k^{2}\right)^{-1}
$$

satisfies

$$
A L-i L A \equiv[A, L]=G^{\prime} \text {. }
$$

Proof: We calculate

$$
\begin{aligned}
& \text { } 4[A, L]\left(L+k^{2}\right)=4\left[A, L+k^{2}\right]\left(L+k^{2}\right)=\left[G^{\prime \prime}-2 G D, L+k^{2}\right] \\
& \therefore=\left[G^{\prime}-2 G D, L\right]=\cdots=4 G^{\prime} D^{2}-\left(G^{\prime \prime}+2 u^{\prime} G\right)=4 G^{\prime}\left(D^{2}+u+k^{2}\right) \\
& =4 G^{\prime}\left(L+k^{2}\right) \text {. }
\end{aligned}
$$

The factor $\left(L+k^{2}\right)$ in the first and in the last expression can be cancelled because it possesses an inverse in the ring of formal power series in $(2 k)^{-1}$

Proposition 1.2: The linear differential operators $A_{n}$ defined by

$$
A=4 \sum_{n=0}^{\infty} A_{n}(-1)^{n}(2 k)^{-2 n-1}
$$

admit the recursive representation

$$
A_{n+1}=4 A_{n} L+2 G_{n} D-G_{n}^{\prime}, \quad A_{0}^{\prime}=0, \cdots A_{1}=D
$$


as.zell as the explicit representation

$$
A_{n+1}=\sum_{m=0}^{n}\left(2 G_{m} D-G_{m}{ }^{\prime}\right)(4 L)^{n-m} .
$$

Each operator $A_{n}$ is formally anti-selfadjoint, i.e. $A_{n}{ }^{*}=-A_{n}$. (Here ${ }^{*}$ means the formal adjoint of a linear differential operator.) Further, there holds

$$
A_{n} L-L A_{n} \equiv\left[A_{n}, L\right]=G_{n}{ }^{\prime} .
$$

Prof : The equations (1.9), (1.11) for the sequences $\left(G_{n}\right),\left(A_{n}\right)$ are equivalent to the equations (1.6), (1.7), for their generating functions $G, A$, respectively. Then (1.10) and $A_{n}+A_{n}{ }^{*}=0$ follow from (1.9) by mathematical induction with respect to $n$. Considering! $L^{*}=L$ and $D^{*}=-D$ we obtain in the induction step $A_{n+1}$ $+A_{n+1}^{*}=\cdots=4\left[A_{n}^{\prime}, L\right]-4 G_{n}^{\prime}=0$

Our introduction of the differential operators $A_{n}$ is a standard one; ef., e.g.', $\mathrm{O}$. I' BogojavienskiJ [15]. A considerably older construction is due to J. L. Burchnall and T. W. CrIAONDY [2]; these authors directly worked with the sequences $\left(G_{n}\right)$, $\left(A_{n}\right)$.

'Now is the time to introduce the Korteiveg-de Vries hicrarchy.

Definition: Let $u=u(x, t)$ and all objects constructed from $u$ depend on the time $t$ as a parameter. The partial differential equation for $u=u(x, t)$ in the Lax 'representation

$$
\frac{\partial L}{\partial t}:=\left[A_{n}, L\right] \quad \because(n=2,3, \ldots)
$$

or in the equivalent function representation

$$
\frac{\partial u}{\partial t}=\frac{\partial}{\partial x} G_{n}[u] \quad(n=2,3, \ldots)
$$

is called $n$-th Korteweg-de Vries equation. The sequence of these equations is called Korteweg-de Vries hierarchy.

Let us recall that two linear differential operators $A, L$ depending on $t$ as a parameter form a Lax pair if

$$
\frac{\partial L}{\partial t}=[A, L] \equiv A L-L A
$$

Here $\partial L / \partial t$ is defined by the Leibniz rule

$$
\therefore \frac{\partial}{\partial t}(L y)=: \frac{\partial L}{\partial t} y+L \frac{\partial y}{\partial t}{ }^{\prime}
$$

Particularly, for $L=D^{2}+u$ the operator $\partial L / \partial t$ is the multiplication by $\partial u$ ! $\partial t$; hence (1.12) and (1.13) are equivalent.

Example: The sequence $\left(G_{n}\right)$ begins as

$$
\begin{aligned}
& G_{0}=1 / 2, \quad G_{1}=u, \quad \dot{G}_{2}=u_{2}+3 u^{2} \\
& G_{3}=u_{4}+10 u u_{2}+5 u_{1}^{2}+10 u^{3} \\
& \stackrel{\prime}{G}_{4}=u_{6}+14 u u_{4}+28 u_{1} u_{3}+21 u_{2}^{2}+70 u^{2} u_{2}+70 u u_{1}^{2}+35 u^{4} \\
& \dot{G}^{2}
\end{aligned}
$$


These expressions are given in $[4,11,14]$ and in other papers. The sequence of differential operators $\left(A_{n}\right)$ begins as

$$
\begin{aligned}
& A_{1}=D_{\wedge}, \quad A_{2}=4 D^{3}+D 3 u+3 u D \\
& A_{3}=16 D^{5}+D^{3} 20 u+20 u D^{3}+D 5\left(3 u^{2}-u_{2}\right)+5\left(3 u^{2}-u_{2}\right) D .
\end{aligned}
$$

These are given in [17] and in other papers.

\section{$\$ 2$ Derivation of the explicit expression}

The time $s$ used in this paragraph has nothing to do with the time $t$ in the Kortewegde Vries hierarchy and is, therefore, notationally distinguished.

Definition: The fundamental solution $K=K\left(x, x_{0}, s\right)$ of the heat equation

$$
\frac{\partial K}{\partial s}=L K \doteq D^{2} K+u K
$$

or, shortly, the heat kernel is defined for $s>0$ and $x, x_{0} \in I$, where $I . \subseteq R$ is some open interval. Moreover, the function

$$
H=H\left(x, x_{0}, s\right)=(4 \pi s)^{1 / 2} \exp \cdot\left(\frac{\left(x-x_{0}\right)^{2}}{4 s}\right) K\left(x, x_{0}, s\right)
$$

is required to admit a smooth extension to $\bar{s} \geqq 0$ such that $H\left(x, x_{0}, 0\right)=1$.

Proposition 2.1: If $u=u(x)$ is defined and smooth in some interval, then there exists a subinterval $I$ and a heat kernel $K=K\left(x, x_{0}, s\right)$ for $x, x_{0} \in I$ and $s>0$. This is uniquely determined, smooth and symmetric in its arguments $x, x_{0}$, that means.

$$
K\left(x, x_{0}, s\right)=K\left(x_{0}, x, s\right)
$$

The corresponding function $H=H\left(x, x_{0}^{\prime}, s\right)$ satisfies

$$
\left[\left(x-x_{0}\right) D+s \frac{\partial}{\partial s}\right] H=s L H .
$$

For the proof of existence, unicity, and of further properties of $K$ we refer to the literature. $[1,12]$. In fact, the construction of the heat kernel works for quite more'general second-order differential operators $L$. In our case, the formula (2.2) - is equivalent to the heat equation (2.1).

The following.proposition is essentially due to H. P. McKéan and P..van MoerBEKE [11]. Our proof is a simplified version of that in [11].

Proposition 2.2: The "diagonal values" $H(x, s):=H(x, x, s)$ obey

$$
\grave{2}\left(2 s \frac{\partial}{\partial s}-1\right) \cdot D H(x, s)=s\left(D^{3}+2 D u+2 u D\right) H(x, s) .
$$

Proof: We apply the operator $D+3 D_{0}$ to

$$
\text { - }\left[\left(x-x_{0}\right) D+s \frac{\partial}{\partial s}\right] H=s L H
$$


and, analogously, the operator $D_{0}+3 D$ to

where

$$
\left[\left(x_{0}-x\right) \dot{D}_{0}+s \frac{\partial}{\partial s}\right] \cdot H=s \dot{L}_{0} H
$$

$$
D=\frac{\partial}{\partial x}, \quad D_{0} \doteq \frac{\partial}{\partial x_{0}}, \quad L=D^{2}+u(x), \quad L_{0}=D_{0}^{2}+u\left(x_{0}\right) .
$$

The second equation-for $H=H\left(x, x_{0}, s\right)$ follows from the first and frow the symmetry in $x, x_{0}$. We add the results and restrict then to the diagonal $x=x_{0}$ of $I \times I$. The identities

$$
\begin{aligned}
& \left(D+3 D_{0}\right)\left(x-x_{0}\right) D+\left(D_{0}+3 D\right)\left(x_{0}-x\right) D_{0} \\
& =-2\left(D+D_{0}\right)+\left(x-x_{0}\right)\left(D^{2}-D_{0}^{2}\right), \\
& \left(D+3 D_{0}\right) D^{2}+\left(D_{0}+3 D\right) D_{0}^{2}=\left(D+D_{0}\right)^{3}
\end{aligned}
$$

are taken into consideration as well as the rules

$$
\left[\left(D+D_{0}\right) y\right](x, x)=d[y(x, x)], \quad\left[\left(D+D_{0}\right)^{3} y\right](x, x)=d^{3}[y(x, x)],
$$

for two-point functions $y=y\left(x, x_{0}\right)$, where $d=d / d x$ denotes, the total derivative with respect to $x$. We arrive at

$$
2\left(2 s \frac{\partial}{\partial s} \div 1\right) d H(x, x, s)=s\left(d^{3}+4 u d+2 u_{1}\right) H(x, x, s),
$$

which is equivalent to $(2.3)$

The following classical construction is connected with the names S. MrNakshIsundaram and A. Pleijel [12]:

Proposition 2.3: Let a sequence of two-point quantities $H_{n}=H_{n}\left(x, x_{0}\right)(n=0,1, \ldots)$ be defined by

$$
\left[\left(x-x_{0}\right) D+n\right] H_{n}=L H_{n-1} \text { for }{ }^{\prime} n \geqq 1, \quad H_{0}=1 .
$$

With this there holds the asymptotic expansion.

$$
H\left(x,-x_{0}, s\right) \sim \sum_{n=0}^{\infty} H_{n}\left(x, x_{0}\right) s^{n} \text { for } s \rightarrow+0 \ldots
$$

For a proof ef. $[1,11,12]$.

In fact, the differèntial-recursion equation system (2.4) has a unique smooth solution $H_{n}=H_{n}\left(x, x_{0}\right),(n=0,1, \ldots)$ in $I \times I, I$ here denoting the domain of definition of $u$, which is recursively given by

$$
H_{n}\left(x, x_{0}\right)=\int_{0}^{1} \lambda^{n-1}\left(L \dot{H}_{n-1}\right)\left(\lambda x+(1-\hat{\lambda}) x_{0}\right) d \lambda \text { for } n \geqq 1 .
$$

This can be directly verified or already follows from classical considerations by J. HADAMARD [6] for more general second-order differential operators $L$. We will call, following [5], the $H_{n}=H_{n}\left(x, x_{0}\right)(n=0,1, \ldots)$ "Hadamard's coefficients".

Proposition 2.4: The one-point quantities

$$
H_{n}{ }^{p}=H_{n}{ }^{p}(x):=\left(D^{p} H_{n}\right)(x, x) \text { for } p, n \geqq 0
$$


derived from the two-point quantities $H_{n}=H_{n}\left(x, x_{0}\right)$ obey the algebraic recursion system

$$
(p+n) H_{n}{ }^{p}=H_{n-1}^{p+2}+\sum_{q=0}^{p}\left(\begin{array}{l}
p \\
q
\end{array}\right) u_{p-q} H_{n-1}^{q} \text { for } n \geqq 1 ; \quad H_{0}^{p}=\delta_{0}{ }^{p} .
$$

As a consequence, each ${H_{n}}^{p}$ for $n \geqq 1$ is a differential polynomial in $u$, that means $a$ polynomial in ' $u, u_{1}, u_{2}, \ldots$ with constant coefficients and without an absolute term.

Proof: We apply $D^{p}=(\partial / \partial x)^{p}$ to both sides of the equation (2.4) and restrict then to the diagonal $x=x_{0}$ of $I \times I$. Throughout the paper $\delta_{q}{ }^{p}$ denotes the Kronecker symbol

Proposition 2.5: The diagonal values of Hadamard's coefficients are proportional to the quantities $G_{n}$ of $\S 1$, more precisely

$$
2(n !) G_{n}[u](x)=(2 n) ! H_{n}(\dot{x}, x) \text { for } n \geqq 0 .
$$

Proof: The equation (2.3) for

$$
\therefore H(x, s)=\sum_{n=0}^{\infty} H_{n}(x, x) s^{n}
$$

implies the differential-recursion system

$$
2(2 n-1) D \dot{H}_{n+1}^{0}=\left(D^{3}+2 u D+2 D u\right) \cdot \dot{H}_{n}{ }^{0}, \quad{ }^{\prime} H_{0}{ }^{0}=1 .
$$

The comparison with

$$
D G_{n+1}=\left(D^{3}+2 u D+2 D u\right) G_{n}, \quad G_{0}=1 / 2
$$

shows (2.6) by mathematical induction. The sequences $\left(H_{n}{ }^{0}\right),\left(G_{n}\right)$ are uniquely determined by the above recursions and by the additional property of being differential polynomials in $u$

In the following, we use the notations

$$
\text { - } u_{0}{ }^{\prime}=u, \quad u_{-1}=0 ; \quad u_{-2}^{\prime}=1, \quad c(p, q)=\left(\begin{array}{l}
p \\
q
\end{array}\right)+\delta_{q}^{p+2}
$$

for integers $p, q \geqq 0$.

Theoren! : There holds for $n \geqq 2$

$$
\begin{aligned}
H_{n}{ }^{p}= & \sum_{q_{1}, q_{3}, \ldots, q_{n}}\left[\left(\dot{q}_{1}+n\right)\left(q_{2}+n-1\right) \ldots\left(q_{n}+1\right)\right]^{-1} \\
& \times c\left(q_{1}, q_{2}\right) c\left(q_{2}, q_{3}\right) \ldots c\left(q_{n-1}, q_{n}\right) \\
& \times u_{q_{1-q},} u_{q_{1-q}} \ldots u_{q_{n-1}-q_{n}} u_{q_{n}},
\end{aligned}
$$

where $q_{\mathrm{l}}=p$ and where the sum runs according to

$$
0 \leqq q_{2} \leqq q_{1}+2,0 \leqq q_{3} \leqq q_{2}+2,::, 0 \leqq q_{n} \leqq q_{n-1}+2:
$$

Corollary 1 : There holds for $n \geqq 1$

$$
H_{n}{ }^{p}=(n !)^{-1}\left(\begin{array}{c}
2 n+p-1 \\
n
\end{array}\right)-1
$$


where the points ... indicate terms in $u$ of lower order and higher degree. Further, there holds for $n \geqq 1$

$$
\begin{aligned}
H_{n}^{p}= & \sum\left[\left(r_{1}+1\right)\left(r_{1}+r_{2}+2\right) \ldots\left(r_{1}+\cdots+r_{n}+n\right)\right]^{-1} \\
& \times\left(\begin{array}{c}
p \\
r_{1}, r_{2}, \ldots, r_{n}
\end{array}\right) u_{r_{1}} u_{r_{1}} \ldots u_{r_{n}}+\cdots
\end{aligned}
$$

where the sum runs over all integers $r_{1}, r_{2}, \ldots, r_{n} \geqq 0$ such that $r_{1}+r_{2}+\cdots+r_{n}=p$.

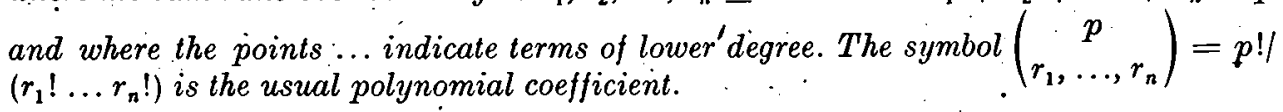

Corollary 2: There holds for $n \geqq 1$

$$
\begin{aligned}
n H_{n}{ }^{0}= & \left.\sum\left[\left(q_{2}+n-1\right)\left(q_{3}+n-2\right) \ldots \hat{\left(q_{n}\right.}+1\right)\right]^{-1} \\
& \times c\left(0, q_{2}\right) c\left(q_{2}, q_{3}\right) \ldots c\left(q_{n-1}, q_{n}\right) u_{-q_{2}} u_{q_{2}-q_{2}} \ldots u_{q_{n-1}-q_{n}} u_{q_{n}},
\end{aligned}
$$

where' the sum runs, over all integers $q_{2}, q_{3}, \ldots, q_{n}$ according to (2.8).

Proofs: With our special notations the recursion system (2.5) can be formally simplified to

$$
\begin{aligned}
& H_{n}^{p}=\sum_{q=0}^{p+2}(p+n)^{-1} c(p, q) u_{p-q} H_{n-1}^{q} \text { for } n=2 \\
& H_{0}{ }^{p}=\delta_{0}^{p}, \quad H_{1}^{p}=(p+1)^{-1} u_{p} .
\end{aligned}
$$

Hence (2.7) follows by mathematical induction with respect to $n$. The first assertion (2.3) of Corollary 1 is better shown by a mathematical induction directly applied to (2.5). The terms of maximal degree in $H_{n}{ }^{p}$ appear for

$$
\begin{aligned}
& r_{n}:=q_{1}-q_{2} \geqq 0, \ldots, r_{2}:=q_{n-1}-q_{n} \geqq 0, r_{1}:=q_{n} \geqq 0, \\
& c\left(q_{1}, q_{2}\right)=\left(\begin{array}{l}
q_{1}^{\prime} \\
q_{2}
\end{array}\right), \quad c\left(q_{2}, q_{3}\right)=\left(\begin{array}{l}
q_{2} \\
q_{3}
\end{array}\right), \ldots \\
& c\left(q_{1}, q_{2}\right) c\left(q_{2}, q_{3}\right) \ldots c\left(q_{n-1}, q_{n}\right)=\left(\begin{array}{c}
q_{1} \\
r_{1}, r_{2}, \ldots, r_{n}
\end{array}\right) .
\end{aligned}
$$

This gives the second assertion (2.10) of Corollary 1. In order to obtain Corollary 2 we have to insert into (2.7) the particular value $p=q_{1}=0$. Our formulas (2.6), (2.11) together give the explicit expression for $G_{n}$ quoted in the introduction

Example: There holds.

$$
\begin{aligned}
& (p+1) H_{1}^{p}=u_{p} \\
& (p+1)(p+2)(p+3) H_{2}^{p}=(p+1) u_{p+2}+(p+3) \sum_{q=0}^{p}\left(\begin{array}{l}
p+1 \\
q+1
\end{array}\right) u_{q} u_{p-q}
\end{aligned}
$$

J..E. LAGNESE [7] explicitly calculated $H_{3}{ }^{p}$ too in this fashion; we omit here. this lengthy expression. 


\section{$\$ 3$ Other properties of the differential polynomials}

The following notions have been introduced in [13] and are useful to describe differential polynomials associated to the Korteweg-de Vries hierarchy.

Definition: A (differential) monomial

$$
c u^{k_{0}} u_{1}^{k_{1}} \ldots u_{p}^{k_{p}}
$$

with integers $p \geqq 0, k_{0} \geqq 0, \ldots, k_{p-1} \geqq 0, k_{p} \geqq 1$ has the order $p$, the degree

$$
d:=k_{0}+k_{1}+\cdots+k_{p},
$$

and the weight

$$
w:=2 k_{0}+3 k_{1}+\cdots+(p+2) k_{p}
$$

A differential polynomial has as its order, degree, weight the maximum of the orders, degrees, weights, respectively, of its monomials. A differential polynomial is called homogeneous if all its monomials have the same weight.

Proposition 3.1 : The differential polynomial $H_{n}{ }^{p}$ for $n \geqq 1$ is homogeneous of weight $2 n+p$, has the degree $n$ and the order $2 n+p-2$. The differential polynomial $G_{n}$ for $n \geqq 1$ is homogeneous of weight $2 n$, has the degree $n$ and the order $2 n-2$.

1 The proof is done by mathematical induction with respect to $n$

The dependence of our differential polynomials on the variable $u=u_{0}$ is fully known.

Proposition 3.2: There holds for $n \geqq 1$

$$
\begin{aligned}
& G_{n}=\sum_{m=0}^{n}\left(\begin{array}{c}
n-1 / 2 \\
n-m
\end{array}\right)(4 u)^{n-m}\left[G_{m}\right]_{u=0}, \\
& H_{n}^{p}=\sum_{m=0}^{n}[(n-m) !]^{-1} u^{n-m}\left[H_{m}^{p}\right]_{u=0} .
\end{aligned}
$$

Proof: We define

$$
\tilde{u}=\tilde{u}\left(x, x_{0}\right)=u(x)-u\left(x_{0}\right), \quad \bar{k}=k\left(1+k^{-2} u\left(x_{0}\right)\right)^{1 / 2}
$$

and indicate objects belonging to $\tilde{u}, \tilde{k}$ by $\sim$. There holds $\tilde{G}=G$ because this generating function depends only on $\tilde{u}+\hat{k}^{2}=u+k^{2}$. Inserting

into

$$
(2 \bar{k})^{-2 n-1}=\sum_{m=0}^{\infty}\left(\begin{array}{c}
n+m-1 / 2 \\
m
\end{array}\right)\left(-4 u\left(x_{0}\right)\right)^{m}(2 k)^{-2(n+m)-1}
$$

$$
\sum_{n=0}^{\infty} \tilde{G}_{n}(-1)^{n}(2 \tilde{k})^{-2 n-1}=\sum_{n=0}^{\infty} G_{n}(-1)^{n}(2 k)^{-2 n-1}
$$

we obtain

$$
G_{n}=\sum_{m=0}^{n}\left(\begin{array}{c}
n-1 / 2 \\
n-m
\end{array}\right)\left(\dot{4} u\left(x_{0}\right)\right)^{n-m} \tilde{G}_{m}
$$

which is equivalent to (3.1). The heat kernel transforms as

$$
K\left(x, x_{0}, s\right)=\mathrm{e}^{u\left(x_{0}\right) s} \tilde{K}\left(x, x_{0}, s\right) .
$$


Herefrom follows, step by step,

$$
\begin{aligned}
& H\left(x, x_{0}, s\right)=\mathrm{e}^{u\left(x_{0}\right) s} \hat{H}\left(x, x_{0}, s\right), \\
& H_{n}\left(x, x_{0}\right)=\sum_{m=0}^{n}[(n-m) !]^{-1} u\left(x_{0}\right)^{n-m} \tilde{H}_{n}\left(x, x_{0}\right), \\
& H_{n}^{p}\left(x_{0}\right)=\sum_{m=0}^{n}[(n-m) !]^{-1} u\left(x_{0}\right)^{n-m} \tilde{H}_{m}^{p}\left(x_{0}\right) .
\end{aligned}
$$

The last formula is equivalent to. (3.2)

Proposition 3.3: There holds for $n \geqq 1$

$$
G_{n}=u_{2 n-2}+\sum_{p+q=2 n-4}\left\{\left(\begin{array}{c}
2 n-2 \\
p+1
\end{array}\right)+(-1)^{p}\right\} u_{p} u_{q}+\cdots,
$$

where the points ... indicate for $n \geqq 3$ terms of degree greater than 2 and of order less than $2 n-5$. As a consequence, we have for the right-hand sides of the Korteweg-de Vries hierarchy

$$
\frac{\partial}{\partial x} G_{n}=u_{2 n-1}+\sum_{p+q=2 n-3}{ }^{\prime}\left(\begin{array}{c}
2 n-1 \\
p+1
\end{array}\right) u_{p} u_{q}+\cdots
$$

The sums in (3.3), (3.4) begin with $p^{\prime}=0$ and end with $q=0$. Further, there holds for $n \geqq 6$

$$
\begin{gathered}
60\left(\begin{array}{c}
2 n \\
n
\end{array}\right)^{-1} G_{n}=30 u^{n}+10\left(\begin{array}{l}
n \\
2
\end{array}\right) u^{n-2} \dot{u}_{2}+3\left(\begin{array}{l}
n \\
3
\end{array}\right) u^{n-3}\left(u_{4}+5 u_{1}{ }^{2}\right) \\
+\quad+6\left(\begin{array}{l}
n \\
4
\end{array}\right) u^{n-4}\left(4 u_{1} u_{3}+3 u_{2}^{2}\right)+110\left(\begin{array}{l}
n \\
5
\end{array}\right) u^{n-5} u_{1}{ }^{2} u_{2} \\
+\quad+75\left(\begin{array}{l}
n \\
6
\end{array}\right) u^{n-6} u_{1}^{4}+\cdots
\end{gathered}
$$

where the points ... indicate terms of degree less than $n-2$. This expression is aiso valid for $n \geqq 0$ if terms with negative powers of $u$ are formally omitted. As, a consequence, we have for the right-hand sides of the Korteweg-de Vries hierarchy

$$
\begin{aligned}
60\left(\begin{array}{c}
2 n \\
n
\end{array}\right)^{-1} \frac{\partial}{\partial x} G_{n}= & 30 n u^{n-1} u_{1}+10\left(\begin{array}{l}
n \\
2
\end{array}\right) u^{n-2} u_{3} \\
& +3\left(\begin{array}{l}
n \\
3
\end{array}\right) u^{n-3}\left(u_{5}+20 u_{1} u_{2}+5 u_{1}{ }^{3}\right) \\
& +12\left(\begin{array}{l}
n \\
4
\end{array}\right) u^{n-4}\left(3 u_{1} u_{4}+5 u_{2} u_{3}\right) \\
+ & +10\left(\begin{array}{l}
n \\
5
\end{array}\right) u^{n-5}\left(23 u_{1}{ }^{2} u_{3}+31 u_{1} u_{2}{ }^{2}\right) \\
& - \\
+ & +960\left(\begin{array}{l}
n \\
6
\end{array}\right) u^{n-6} u_{1}{ }^{3} u_{2}+\ldots
\end{aligned}
$$


Proof: Let $L_{n}$ denote the linear part of. $G_{n}$ and $Q_{n}$ the quadratic part, respectively. These obey the differential-recursion system

$$
\begin{aligned}
& L_{n+1}=D^{2} L_{\dot{n}}, \quad L_{1}=u, \\
& D Q_{n+1}=D^{3} Q_{n}+2(D u+u D) L_{n}, \quad Q_{2}=3 \dot{u^{2}}
\end{aligned}
$$

Mathematical induction shows

$$
L_{n}=u_{2 n-2}, \quad Q_{n}=\sum_{p+q=2 n-4}\left\{\left(\begin{array}{c}
2 n-2 \\
p+1
\end{array}\right)+(-1)^{p}\right\} u_{p} u_{q}
$$

All other terms in $G_{n}$ must have an order less than $2 n-5$. Considering

$$
4^{n-m}\left(\begin{array}{c}
n-1 / 2 \\
n-m
\end{array}\right)=\left(\begin{array}{c}
2 n \\
n
\end{array}\right)\left(\begin{array}{c}
n \\
m
\end{array}\right)\left(\begin{array}{c}
2 m \\
m
\end{array}\right)^{-1}
$$

we rewrite (3.1) as

$$
\left(\begin{array}{c}
2 n \\
n
\end{array}\right)^{-1} G_{n}=\sum_{m=0}^{n}\left(\begin{array}{c}
2 m \\
m
\end{array}\right)^{-1}\left(\begin{array}{c}
n \\
m
\end{array}\right) u^{n-m}\left[G_{m}\right]_{u=0}
$$

and insert herein

$$
\begin{aligned}
& G_{0}=1 / 2, \quad G_{1}=u, \quad G_{2}=u_{2}+3 u^{2}, \\
& {\left[G_{3}\right]_{u=0}=u_{4}+5 u_{1}^{2}, \quad\left[G_{4}\right]_{u=0}=28 u_{1} u_{3}+21 u_{2}^{2}+\cdots,} \\
& {\left[G_{5}\right]_{u=0}=462 u_{1}^{2} u_{2}+\cdots, \quad\left[G_{6}\right]_{u=0}=1155 u_{1}^{4}+\cdots,}
\end{aligned}
$$

where the points indicate terms which do not contribute to (3.5). The result (3.3) is due to P. B. GrKKEY [4], who established it by a quite other method, not being aware of the relation to the Korteweg-de Vries hierarchy

More properties of the sequence $\left(G_{n}\right)$ can be found in the literature $[3,8-1 \dot{0}, 16$, 17]. In [16] it is shown that

$$
\frac{\partial}{\partial u} G_{n+1}=\frac{\delta}{\delta u} G_{n+1}=2(2 n+1) G_{n}
$$

where

$$
\frac{\delta}{\delta u}:=\sum_{p \geq 0}(-D)^{p} \frac{\dot{\partial}}{\partial u_{p}}
$$

denotes the variational or Euler-Lagrange derivative. For each couple.of integers $m, n \geqq 1$ the quantity $G_{m}$ is a conserved density of the $n$-th Korteweg-de Vries equation $u_{t}=G_{n}[u]_{x}[3,9,10,16]$.

We will finish with a formula which merely follows from the symmetry property $H_{n}\left(x, x_{0}\right)=H_{n}\left(x_{0}, x\right)$. The proof runs along the lines of [7] and will, therefore, be omitted.

Proposition 3.4: There holds for integers $n \geqq 1, k \geqq 0$

$$
2 H_{n}{ }^{2 k+1}=D \sum_{q=0}^{2 k}\left(\begin{array}{c}
2 k+1 \\
q+1
\end{array}\right)(-D)^{q} H_{n}^{2 k-q} .
$$

As a consequence, $H_{n}{ }^{2 k+1}$ can be expressed as a linear differential expression in $H_{n}{ }^{0}$, $H_{n}{ }^{2}, \ldots, H_{n}{ }^{2 k}$. 
The relevance of the quantities $G_{n}(n=1,2, \ldots)$ for the theory of Huygens' principle is discussed in the paper [14]. If the wave-like operator in $2 m+2$ dimensions

$$
L-\Delta \equiv \frac{\partial^{2}}{\partial x^{2}}+u(x)-\sum_{i=2}^{2 m+2} \frac{\partial^{2}}{\partial x_{i}^{2}}
$$

is a Huygens-type one, then $G_{n}[u]=0$ for $n \geqq m$.

\section{REFERENCES}

[1] Berger, M., et al.: Le Spectre d' une Varieté Riemannienne. Berlin: 'Springer-Verlag 1971.

[2] Burchnale, J. L., and T. W. Chaundy: Commutative ordinary differential operators. Proc. London Math. Soc. (2) 21 (1922), 420-440.

[3] Gardner, C. S., et al.: Korteweg-de Vries Equation and Generalizations VI. Commun. Pure Appl. Math. 27 (1974), 97-133.

[4] GLKey, P. B.: Recursion relations and the asymptotic behaviour of the eigenvalues of the Laplacian. Compos. Math. 38 (1979), 201-240.

[5] Güмтнer, P.: Hadamard coefficients and curvature for the de Rham complex. Wiss. Z. Karl-Marx-Univ. Leipzig, Math.-Naturw. R. 99 (1980), $65-70$.

[6] Hadamako, J.: Lectures on Cauchy's Problem. New Haven: Yale Univ. Press 1923.

[7] Lagsfse, J. E.: A New Differential Operator of the Pure Wave Type. J. Diff. Equ. 1 (1965), $171-187$.

[8] LAx, P. D.: Integrals of Nonlinear Equations of Evolution and Solitary Waves. Commun. Pure Appl. Math. 21 (1968), 467-490.

[9] Lax,.P. D.: Periodic Solutions of the KdV Equation. Commun. Pure Appl. Math. 28 (1975), $141-188$.

[10] Lax, P. D.: Almost Periodic Solutions of the KdV Equation. SIAM Review $1 S$ (1976), $351-375$.

[11] McKean, H. P., and P. Van Monrbeke: The spectrum of Hill's equation. Invent. Math. 30 (1975), $217-274$.

[12] Munakseisundaram, S., and A. Pleijel: Some properties of the eigenfunctions of the Laplace operator on Riemannian manifolds. Can. J. Math. 1 (1949), 242-256.

[13] Mrura, R. M., et al.: Korteweg-de Vries Equation and Generalizations II. J. Math. Phys. 9 (1968), 1204-1209.

[14] Schmmina, R.: Korteweg-de Vries-Hicrarchie und Huygenssches Prinzip. Preprint. Dresdener Seminar zur Theor. Physik Nr. 26, 1986.

[15] Богоявленский, О. И:: Об интегралах высших стационарных уравнени í собственных числах операторов Хилла. Функц. анализ и его прил. 10 (1976) 2, $9-12$.

[16] Гкльфанд, И. М., и Л. А. Диний: Асимптотика резольвеиты штурма-лиувиллевских уравнений и алгебра уравнений Кортевега-де Фриза. Успехи мат. наук 30 (1975) 5, 67-100.

[17] Захагов, В. Е., и др.: Теория солитонов. Москва: Изд-во Наука 1980.

[18] МАрченко, В. А.: Операторы ІШтурма-Јиупилля и их приложения. Киев: Наукова Думка 1977.

[19] Новиков, С. П.: Периодическая задача уравнения Кортевега-де Фриза. Функц. анализ и его прил. 8 (1974) $3,54-66$.

Manuskripteingang: 13.04.1987

VERFASSER :

Doz. Dr. sc. RiIner Schimming

Sektion Mathematik der Ernst-Moritz-Arndt-Universität

Friedrich-Ludwig-Jahn-Str. 15 a

DDR - 2200 Greifswald 